\title{
EFFECTIVE SYSTEM FOR AUTOMATIC BUNDLE BLOCK ADJUSTMENT AND ORTHO IMAGE GENERATION FROM MULTI SENSOR SATELLITE IMAGERY
}

\author{
A.Akilan ${ }^{\text {a, }}{ }^{*}$, V.Nagasubramanian, AnkitChaudhry, D.RajeshReddy, D.SudheerReddy, R.UshaDevi, T.Tirupati, P.V.Radhadevi, \\ GeetaVaradan.
}

Advanced Data Processing Research Institute

Dept. of Space, Manovikas Nagar P.O., Secunderabad-9

aakilan@adrin.res.in

\begin{abstract}
:
Block Adjustment is a technique for large area mapping for images obtained from different remote sensingsatellites. The challenge in this process is to handle huge number of satellite imageries from different sources with different resolution and accuracies at the system level. This paper explains a system with various tools and techniques to effectively handle the end-to-end chain in large area mapping and production with good level of automation and the provisions for intuitive analysis of final results in 3D and 2D environment. In addition, the interface for using open source ortho and DEM references viz., ETM, SRTM etc. and displaying ESRI shapes for the image foot-prints are explained. Rigorous theory, mathematical modelling, workflow automation and sophisticated software engineering tools are included to ensure high photogrammetric accuracy and productivity. Major building blocks like Georeferencing, Geo-capturing and Geo-Modelling tools included in the block adjustment solution are explained in this paper. To provide optimal bundle block adjustment solution with high precision results, the system has been optimized in many stages to exploit the full utilization of hardware resources. The robustness of the system is ensured by handling failure in automatic procedure and saving the process state in every stage for subsequent restoration from the point of interruption. The results obtained from various stages of the system are presented in the paper.
\end{abstract}

\section{INTRODUCTION}

There is always a trade-off in remote sensing satellite imagery between spatial resolution and the ground coverage. In order to acquire the largest possible coverage, small overlapping satellite images are often used for satellite triangulation. Hence, the traditional 3-D bundle adjustment as used in aerial photogrammetry may not be directly employed for orientation modelling. A public domain digital elevation model like SRTM can be used for weakly convergent images as explained by Liang-Chien Chenet.al, 2008. In the context of increased availability of high resolution images from different satellites, intensive demand in photogrammetry for large area mapping from available images has become inevitable. Block adjustment provides solution to the problem with minimal control points across bundle of images of same or different spatial resolution captured with different geometry..The advantages of the block bundle adjustment over the independent adjustment (as explained by Toutin, 2003) are

- The number of ground control points (GCPs) is largely reduced

- All image geometric models are computed together

- A homogeneous digital elevation model (DEM) can be generated when the block has a full and good stereo coverage

- Abetter relative accuracy between the images can be obtained

- A more homogeneous and accurate ortho-mosaic over large area can be obtained and

- A homogeneous GCP network for future geometric processing can be generated

At ADRIN, rigorous geometric model combining the principles of photogrammetric collinearity equations was originally developed for SPOT-1 [Radhadevi et al., 1994], and further adapted to multi-sensor geometries from IRS-1C/1D to Cartosat2 [Radhadevi, 2013]. Due to its simple implementationand standardization, the RFM have been widely used in the remote sensing community. Rational Function Model was also developed for different sensors [Nagasubramanian et.al, 2007]. We have further extended these models and developed a full-fledged system for Bundle block adjustment. The major building blocks of the proposed bundle block adjustment (BBA) system are described in this paper.Envisaging framework/system for automatic block adjustment for large number of satellite imagery is a challenging task as its workflow involves many processes in which each one is complex on its own. The workflow of the schema is shown in Figure 1.

\section{BLOCK CONSTRUCTION}

Images captured with along and across track overlap are well suited to process in block adjustment. As it is invariant to spatial resolution and orientation, imagery from various satellites can also be submitted for adjustment. The system picks up the images from specified database/file system and to the specified sensors/satellites over an area selected by the user from the coverage plots. For the purpose, the image header or the name itself is supposed to contain the details of satellite/sensor. Overlap information is obtained based on the system level error of imagery. Block comprises of imagesconnected from one to other by overlap. Automatic overlap computation and sorting method is explained by Sudheer Reddy et.al, 2014.Block finalizing is the initial task before proceeding to subsequent adjustment process steps. The images can be overviewed for cloud, water-body and other interests before finalizing. Homogeneous block contains images captured from same satellite/sensor while images from different sources constitute a block is called heterogeneous block.The BBA software stores and displays the complete details such as sensor name, resolution, corner coordinates and overlap-percentage with adjacent images etc., of each image in a block.

* corresponding author 


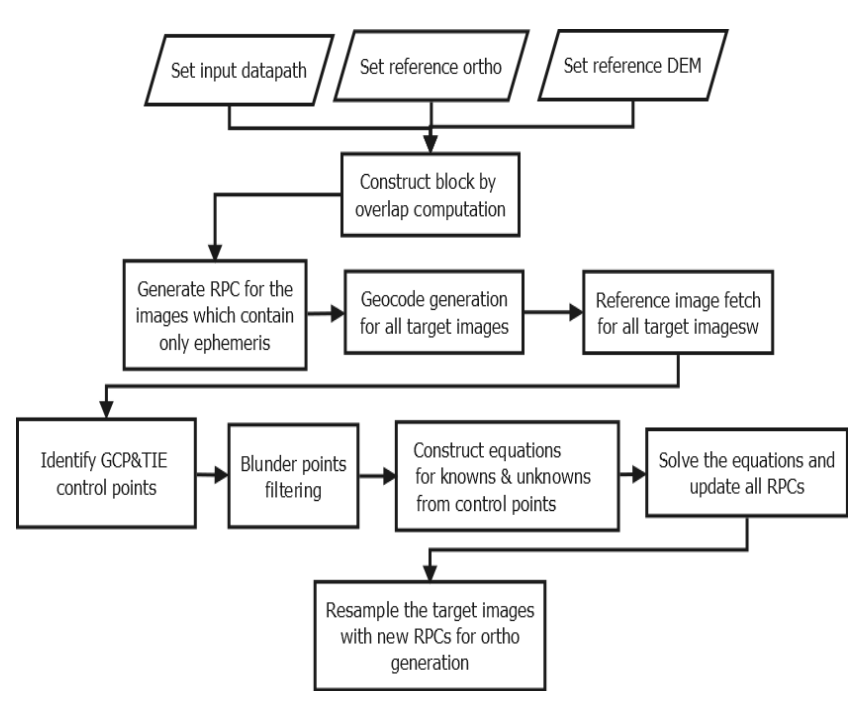

Figure 1. Block adjustment workflow

\section{REFERENCE IMAGE CONFIGURATION}

Ortho-rectified products and Digital Elevation Model (DEM) references are essentially aid to ensure location accuracy of data sets. The Landsat Enhanced Thematic Mapper (ETM), Landsat Data Continuity Mission (Landsat 8), Google maps/ortho photos, Shuttle Radar Topography Mission (SRTM), ASTER Global DEM are such globally available references can be utilized in any planimetry error correction. Other high accuracy references like Cartosat-1 ortho images/DEMs, aerial orthos/DEMs as well as surveyed controls also can be used as references in BBA.The proposed BBA software system extents its interface to fully exploit them along the references generated in local. Required references to the bundle images are picked automatically from the specified database/file system. Projection, datum and geoidal/ellipsoidal transformations for the references are done internally as a part of the process. Addition of any further references is configurable from external file to the software without any internal modification. This capability of the system ensures that the quality of the products improves as availability of better references over a period.

\section{RATIONAL POLYNOMIAL COEFFICIENT GENERATION}

Few satellite agencies give RPCs directly along with image data whereas others provide ephemeris and attitude information. For Indian Remote Sensing Satellites (IRS), we have developed rigorous sensor model. Using the model, a grid file is generated which in turn used for RPC generation.

Rational Polynomial Coefficient (RPC)/Rational Functional Model (RFM) is the easiest way to handle image geometry derived from physical sensor model when block of images are processed. The proposed system uses RPC to avoid complexity in directly dealing with rigorous sensor model (RSM). Initial system level RPCs are generated individually for each image from their respective RSM model. This process is done in parallel for multi core system as each generation is independent of others; thereby rest of all processes is carried out using RPCs only. Using RPC has an advantage because users are not required to know the specific information on the satellite sensors. Thus, it is specifically useful while handling multisensor data out of which few of them directly come along with RPC and remaining with ephemeris.
The basic RFM equation in the forward projection form, i.e., a ground coordinate to the image point coordinate, is expressed as Eq.1.

A rational function model can be represented as the quotient of two polynomials, which describe the geometrical relationship between object and image co-ordinates. It is as follows.

$$
\begin{gathered}
l=\frac{p_{1} X, Y, Z}{p_{2} X, Y, Z} \\
s=\frac{p_{3} X, Y, Z}{p_{4} X, Y, Z}
\end{gathered}
$$

$p_{i}$ are third order polynomials.

$$
\begin{array}{rl}
p_{i} X, Y, Z= & 3 \\
& 3 a_{i j k} X_{i} Y_{j} Z_{k} \\
i=0 & j=0 \\
& =a_{0}+a_{1} X+a_{2} Y+a_{3} Z \\
& +a_{4} X Y+\cdots+a_{19} Z^{3}
\end{array}
$$

whereais are polynomial coefficients and $\mathrm{X}, \mathrm{Y}, \mathrm{Z}$ are normalized object co-ordinates. This may be in spherical or Cartesian co-ordinate system. $l, s$ are normalized image coordinates. Original values of image and ground co-ordinates are offset and scaled to fit between -1.0 to +1.0 .

For the given ground point coordinates, the corresponding image coordinates $(1, s)$ can be computed .RPC models transform three-dimensional object-space coordinates into two-dimensional image space coordinates. RPCs provide a simple and accurate means of Interior Orientation communicating camera object-image relationship from image RPCs have been successfully used for the terrain extraction, ortho rectification, and feature extraction tasks.

\section{AUTOMATIC GROUND CONTROL AND TIE POINTS IDENTIFICATION}

Ground control points are identified on reference to target images in order to achieve good absolute accuracy.. Tie points are identified between bundle images on overlapping region to ensure continuity across them. Initial feature points are collected using forstner operator and subsequently submitted for normalized cross correlation (NCC) to obtain correspondence. The entire matching is done automatically by taking guidance from system level error present in the data sets. For that purpose each date set is geocoded. During the process, the target image and its corresponding reference image are brought to the same scale to facilitate auto matching. This module is made as plug-in type in order to accommodate any robust matching procedure such as SIFT, SURF, ORB etc., in future without affecting rest of the system. Ground surveyed control points are more accurate than any reference in public. Easiest interface present in the system to directly ingest surveyed points beside the display of photographs of the location from various sources. 


\section{DATA ERROR MINIMIZATION AND BLUNDER REMOVAL}

The control points acquired from matching function is based on system level knowledge of the data sets. The images to matching function may possibly be captured in multi temporal conditions, seasons. So the control points extracted at the first level need not be as accurate as expected. Inaccurate points without any filtering mechanism lead to error in planimetry and feature continuity between adjacent images as well. The BBA system employs multi-level robust weeding out mechanism to ensure that points chosen are well accurate in sub-pixel level. In the first level, spurious points are eliminated which are having errors above 3-sigma over physical sensor model respect to data. Second level is based on histogram variation around the points of interest using mutual information (MI) procedure. To meet the sub-pixel level accuracy Random Consensus (RANSAC) method is applied with sensor model (RSM). As all these levels of filtering are not mandatory for all sensor data, user is provided with the option to enable/disable secondary and tertiary level filtering.

\section{BLOCK ADJUSTMENT}

Once all the control and tie points pass through weeding-out process, adjustment procedure in invoked to establish geometric relationship between the target images and between target images and respective ground. Single least square adjustment (LSQ) is applied over the equations generated from ground control and tie points for error minimization well within the threshold. As the adjustment is basic intention of BBA software, the workflow has been broken into two. Either the user can opt for automation till bundle adjustment or till end product generation, such as ortho-rectification, mosaic, merging or coregistration. Map based (Survey Of India scale) and interested area level product generation are also possible from the system. Since the tie points tags the pixels across the multi-spectral bands and between the images, it indirectly addresses the preliminary geometry requirements for band-to-band registration, mosaic and merging.

\section{ERROR ANALYSIS}

In any photogrammetry, error evaluation, analysis and correction are essential work to adjust the input parameters to meet the error budget. Error plot, error log and error statistics are the addition details as result of the bundle adjustment process. Error plot is a visual display of error trend of each GCP, tie and check points over image coverage. Root Mean Square Error (RMSE) is also part of this plot. Once adjustment is done, user can observe through all these details and if needed, the points can be added/removed (erroneous) from adjustment to well improve the accuracy of final product.

\section{AUTO PROCESS FAILURE MANAGEMENT}

Usually the content of the images plays vital role in most of the image processing algorithms. Most cloud cover, water body and long gap in multi-temporal images are some of them can directly lead to failure in automatic feature detection and matching methods. In the proposed BBA framework, such data sets are brought to the notice of user with display. Thereby, user can manually identify some of guidance points (minimum three) and submit back for automatic matching procedure for subsequent GCP and tie points identification. While manual identification is on, other data sets in the bundle proceeds in background till one step before the adjustment procedure.

\section{DISPLAY UTILITY}

Block adjustment framework requires display tool to check the quality of the products generated from the process with visual perception. It creates common canvas of specified projection in order to display block of images to their respective geographic locations. It helps to verify the continuity between the images visually. The proposed system consists of such tool with sufficient number of image enhancement functionalities built-in such as pan, zoom-in, zoom-out, stretch, histogram adjustment etc., In addition, image blending and swiping are the two superior features that are part of the utility to check the pixel to pixel tagging among the images. As the display tool is made component object model paradigm, it is highly modular and portable across different application independent of platform. The utility has been developed with memory mapping technique offered by operating system to handle large sized images that do not fit directly into physical memory.

\section{3D VISUALIZATION TOOL}

Photogrammetry and 3D visualization are inseparable in order to deliver effective data products for planning and other interactive analysis. Large area mapping involves DEM as reference in bundled product generation chain. The BBA software allows the user to drape the DEM over ortho rectified images to get seamless 3D perception of the block. This features to study and analyse the effect of elevation over regions of interests. 3D visualization tool for the framework has been built using open scene graph (OSG) library. OSG is an open source high performance 3D graphics toolkit written in standard $\mathrm{C}++$ and OpenGL runs on all platforms. It is widely used in fields such as visual simulation, games, virtual reality, scientific visualization and modelling [http://www.openscenegraph.org/].

\section{PERFORMANCE IMPROVEMENT AND RESOURCE UTILIZATION}

The possibilities for improving performance in the various stages of the process are well exploited to work efficiently on multi code machines. Especially in the following phases of the chain as they are specific to each dataset,

- Image overview tile generation

- $\quad$ RPC generation from rigorous sensor modelling

- Geocoding and reference image generation

- GCP generation

- Stereo matching algorithm

- Forstner points generation

- Usage of High performance C++ library (Eigen) for least square adjustment process (LSQ)

- Final ortho product generation

- Input/output Grid generation

- Image Resampling 


\section{RESULTS}

The following,Figure 2, are some of the results generated over Indian region by combining data sets from differentsatellite and sensors such as Cartosat-1, Resourcesat2-LISS4/AWiFS, IRSP6 etc., with the time line in Table 1. More evaluations are done with BBA software and results are presented by Sujatha et.al, 2014.
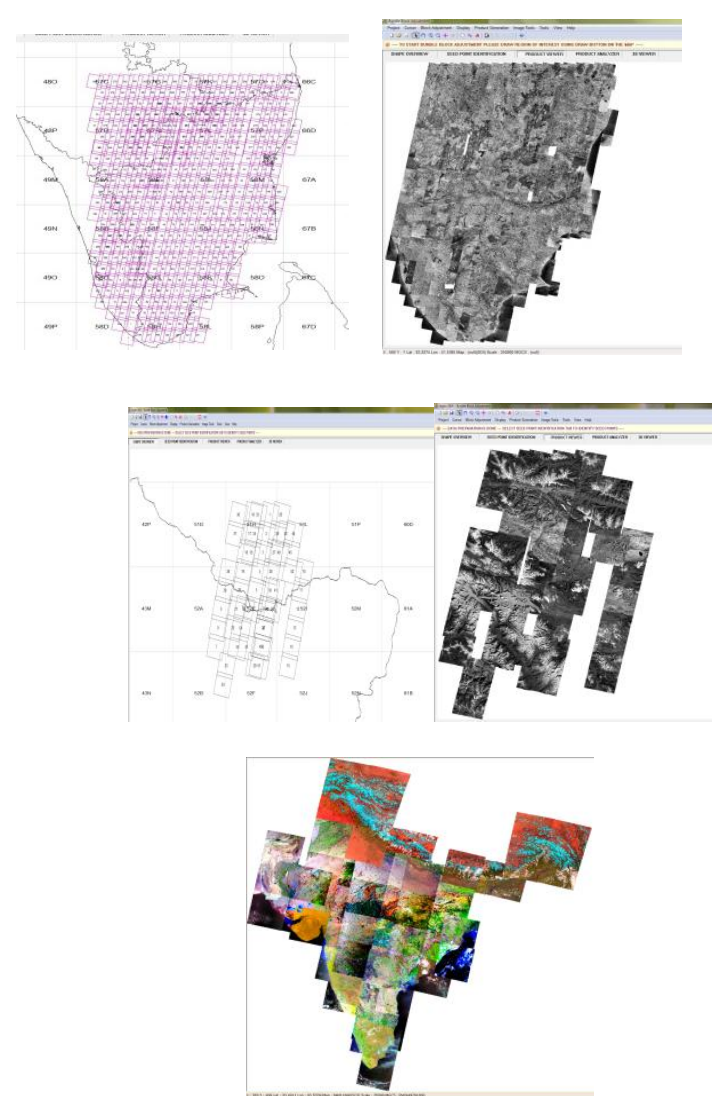

Figure 2. Sample bundle adjusted ortho products results on Indian region

\begin{tabular}{|l|l|l|}
\hline $\begin{array}{l}\text { No. of } \\
\text { Scenes }\end{array}$ & System Configuration & $\begin{array}{l}\text { Total time } \\
\text { taken } \\
\text { (approx.) }\end{array}$ \\
\hline 55 & $\begin{array}{l}\text { DELL Precision T7600 } \\
\text { Processor: Intel (R) Xeon (R) } \\
\text { CPU E5-2650 @ 2.00 GHz (2 } \\
\text { processors)Windows-7, 64 - bit } \\
\text { OS, SP-1RAM : 16 GB }\end{array}$ & 1 hour \\
\hline
\end{tabular}

Table 1. Time taken for automatic block adjustment and ortho generation

\section{Conclusion}

This paper describes the features and development strategies of a Large Area Multi-sensor adjustment and Production System (BBA).The proposed software BBA significantly improves the geometric consistency between overlapping images with respect to the individual adjustment.End-to-end chain from GCP identification to product generation is automatic. All the tests performed demonstrated that the system is stable and the software is robust.

\section{REFERENCES}

D Sudheer Reddy, T Krishna Sumanth, P V Radhadevi, J Saibaba, GeetaVaradan "Algorithms for Automatic Separation and Indexing of image footprints in Satellite Bundle Block Adjustment", to appear in ISRS

Sujata Ghosh*, P S Bhawani Kumar, V.Nagasubramanian, P V Radhadevi, V Srinivas, J Saibaba, Geeta Varadan,2014 Geometric quality assessment of bundle block adjusted multisensor satellite imageries, to appear in the Proceedings of ISPRS,2014,Hyderabad, India

P V Radhadevi,2013.GeometricModeling for High Resolution Indian Remote Sensing Satellite Sensors, IEEE journal of selected topics in applied earth observations and remote sensing, vol. 6, no. 3, june 2013

Liang-Chien Chen, Tee-Ann Teo, and Chao-Yuan Lo,2008. Elevation-controlled block adjustment for weakly convergent satellite images, The International Archives of the Photogrammetry, Remote Sensing and Spatial Information Sciences. Vol. XXXVII. Part B1. Beijing 2008

Nagasubramanian V., Radhadevi P.V.,Ramachandran R. and Krishnan R.,2007. Rational function model for sensor orientation of IRS-P6 Liss-4 imagery.The Photogrammetric Record, 22(120),pp 309-320,2007

Thierry Toutin, 2003. Block Bundle Adjustment of Landsat 7 ETM Images over Mountainous Areas, Photogrammetric Engineering \& Remote Sensing Vol. 69, No. 12, December 2003, pp. 1341-1349,2003

P. V., Radhadevi, T. P. Sasikumar and R. Ramachandran. Orbit Attitude Modelling and derivation of Ground Coordinates from SPOT stereo pairs, ISPRS Journal of Photogrammetry \& Remote Sensing, vol. 49, no. 4, pp. 2229, 1994. 